\title{
Ordered Nanostructure Enhances Electrocatalytic Performance by Directional Micro-Electric Field
}

Qing-Xia Chen, ${ }^{\mathrm{a},+}$ Ying-Huan Liu, ${ }^{\mathrm{b},+}$ Xiao-Zhuo Qi, ${ }^{\mathrm{c}}$ Jian-Wei Liu, ${ }^{*, \mathrm{a}}$ Hui-Jun Jiang,, ,b

Jin-Long Wang, ${ }^{\mathrm{a}}$ Zhen He, ${ }^{\mathrm{a}}$ Xi-Feng Ren, ${ }^{\mathrm{c}}$ Zhong-Huai Hou, ${ }^{\mathrm{b}}$ and Shu-Hong $\mathrm{Yu}^{*, \mathrm{a}}$

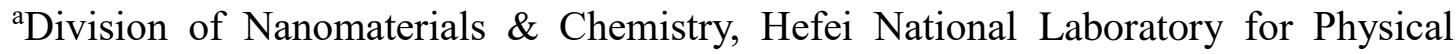
Sciences at the Microscale, CAS Center for Excellence in Nanoscience, Hefei Science Center of CAS, Collaborative Innovation Center of Suzhou Nano Science and Technology, Department of Chemistry, University of Science and Technology of China, Hefei 230026, China. bepartment of Chemical Physics \& Hefei National Research Center for Physical Sciences at Microscales, iChEM, University of Science and Technology of China, Hefei 230026, China. 'Synergetic Innovation Center of Quantum Information \& Quantum Physics, Key Laboratory of Quantum Information, University of Science and Technology of China, Hefei 230026, China.

\section{Contents}

\section{Supporting Experimental Section}

Synthesis of PtTe NWs.

Synthesis of PtPdTe NWs.

Synthesis of PtPdRuTe NTs.

Synthesis of Pt NPs.

Synthesis of Ru@Pt NPs.

Synthesis of Pt NFs.

Synthesis of PtPd NFs.

\section{Supplementary Notes}

Note S1. Details of the kinetic model.

Note S2. Details of numerical simulation.

Note S3. Details of the exposed avaliable surface area simulation.

Note S4. Determination of orientational parameter.

\section{Supplementary Figures}


Figure S1. Morphological and structural characterizations of the Pt NTs.

Figure S2. Optical photograph of glass substrates with various areas and detachable glassy carbon electrode.

Figure S3. Schematic illustrations and result of the small angle X-ray diffraction (SAXRD) measurement.

Figure S4. Polarized light transmission spectrum tests.

Figure S5. Methanol oxidation reaction (MOR) measurements of ordered and disordered Pt NTs nanostructures.

Figure S6. MOR performances of Pt NTs model catalyst recorded with different electrochemical parameters.

Figure S7. Statistical analysis of the exposed available surface areas in different patterns. Figure S8. MOR performances of Pt NTs model catalyst recorded with different rotating speeds applied.

Figure S9. Movement of $\mathrm{CH}_{3} \mathrm{OH}$ jet in electrostatic field.

Figure S10. The numerical simulation setup.

Figure S11. Kinetic simulation results of Pt NTs for MOR.

Figure S12. Representative TEM images of other one-dimensional (1D) Pt-based multicomponent nanostructures.

Figure S13. XRD pattens of 1D Pt-based multicomponent nanostructures.

Figure S14. Ordering determination of ordered PtTe monolayer.

Figure S15. Ordering determination of ordered PtPdTe monolayer.

Figure S16. Ordering determination of ordered PtAuTe monolayer.

Figure S17. Ordering determination of ordered PtPdRuTe monolayer.

Figure S18. MOR performances of other 1D Pt-based multicomponent nanostructures.

Figure S19. Morphological and structural characterizations of Pt-based NPs.

Figure S20. MOR performances of Pt-based NPs.

Figure S21. Morphological and structural characterizations of Pt-based NFs.

Figure S22. MOR performances of Pt-based NFs.

Figure S23. Kinetic simulation results of Pt NPs for MOR.

Figure S24. Kinetic simulation results of Pt NFs for MOR.

Figure S25. FAOR measurements of ordered and disordered Pt NTs monolayer.

Figure S26. Kinetic simulation results of Pt NTs for formic acid oxidation reaction (FAOR).

\section{Supplementary Tables}

Table S1. Component contents in Pt-based nanostructures.

Table S2. Summary of literature catalytic parameters of various Pt-based MOR catalysts.

Table S3. Selection of the parameters in MOR.

Table S4. Selection of the parameters in MOR of OD particles.

Table S5. Selection of the parameters in MOR of 2D films.

Table S6. Selection of the parameters in FAOR.

\section{Supporting Experimental Section}


Synthesis of PtTe NWs. PtTe NWs were synthesized similar to Pt NTs just halving the dose of Pt precursor. After 0.05 mmol as-synthesized Te NWs was collected with sufficient acetone and redispersed in ethylene glycol (EG), $0.05 \mathrm{mmol}$ of $\mathrm{H}_{2} \mathrm{PtCl}_{6}$ dissolved in EG was added. After sufficiently mixed by stirred vigorously for $10 \mathrm{~min}$, the final solution was shaken at a rotation rate of $260 \mathrm{rpm}$ by an Innova 40 Benchtop Incubator Shaker for $13 \mathrm{~h}$ at $50{ }^{\circ} \mathrm{C}$. Finally, the products were washed several times with deionized water (DIW) and ethanol.

Synthesis of PtPdTe NWs. PtPdTe NWs were synthesized similar to PtTe NTs with small modifications. $0.1 \mathrm{mmol}$ of $\mathrm{H}_{2} \mathrm{PtCl}_{6}$ dissolved in $\mathrm{EG}$ was replaced with 0.17 mmol of $\mathrm{H}_{2} \mathrm{PtCl}_{6}$ and $0.17 \mathrm{mmol}$ of $\mathrm{PdCl}_{2} \mathrm{EG}$ dispersions.

Synthesis of PtPdRuTe NTs. The preparation of PtPdRuTe NTs through a galvanic displacement was described by our group recently ${ }^{1}$. In brief, the mixture of $0.18 \mathrm{mmol}$ of Te NWs and $0.15 \mathrm{mmol}$ of $\mathrm{RuCl}_{3}$ was refluxed under magnetic stirring for $1 \mathrm{~h}$ at 190 ${ }^{\circ} \mathrm{C}$. Subsequently, $75 \mu \mathrm{mol}$ of $\mathrm{K}_{2} \mathrm{PtCl}_{6}$ and $75 \mu \mathrm{mol}$ of $\mathrm{K}_{2} \mathrm{PdCl}_{4}$ were added dropwise into the solution above for another $1 \mathrm{~h}$. Finally, the products were washed several times with DIW for further characterizations and measurements.

Synthesis of Pt NPs. Pt NPs were prepared according to a procedure reported already with some modifications ${ }^{2}$. Typically, $84 \mathrm{mg}$ of $\mathrm{K}_{2} \mathrm{PtCl}_{6}$ and $60 \mathrm{mg}$ of PVP were dissolved thoroughly in $40 \mathrm{~mL}$ of EG. And then, the solution was refluxed for $1 \mathrm{~h}$ at $200{ }^{\circ} \mathrm{C}$ under magnetic stirring and nitrogen protection. Finally, the precipitates were washed several times with anhydrous acetone for further characterizations and measurements.

Synthesis of Ru@Pt NPs. Modifications have been made with reported literatures in the preparation of Ru@Pt NPs ${ }^{2-3}$. Briefly, the synthesis of Ru core was on the basis of a reflux method. $37.7 \mathrm{mg}$ of $\mathrm{RuCl}_{3}$ and $0.83 \mathrm{~g}$ of PVP were dissolved adequately in 150 $\mathrm{mL}$ of EG. The mixture was refluxed for $3 \mathrm{~h}$ at $200{ }^{\circ} \mathrm{C}$ under magnetic stirring and nitrogen protection. The products were allowed to cool to room temperature, to which $42 \mathrm{mg}$ of $\mathrm{K}_{2} \mathrm{PtCl}_{6}$ was added. The formed solution was processed for another reflux at 
$200{ }^{\circ} \mathrm{C}$ for $1.5 \mathrm{~h}$. Finally, the precipitates were washed several times with anhydrous acetone for further characterizations and measurements.

Synthesis of Pt NFs. Pt NFs were obtained through a reported procedure with some modifications ${ }^{4}$. Typically, $160 \mathrm{mg}$ of PVP, $100 \mathrm{mg}$ of benzoic acid and $10 \mathrm{mg}$ of $\mathrm{Pt}(\mathrm{acac})_{2}$ were dissolved in $5 \mathrm{~mL}$ of benzyl alcohol, followed by ultrasonic dispersing for $20 \mathrm{~min}$. Thereafter, the resulting mixture was refluxed for $7 \mathrm{~h}$ at $170{ }^{\circ} \mathrm{C}$. The asprepared products were centrifugation and purified with anhydrous acetone for further characterizations and measurements.

Synthesis of PtPd NFs. A similar procedure to that of Pt NFs was conducted to prepare PtPd NFs. The solution before refluxing in preparation of Pt NFs was added with another $7.7 \mathrm{mg}$ of $\mathrm{Pd}(\mathrm{acac})_{2}$.

\section{Supplementary Notes}

\section{Details of the kinetic model.}

In this kinetic model, the reversible electrocatalytic oxidation reactions are

$$
R \underset{k r}{\stackrel{k r}{\rightleftharpoons}} 0,
$$

To start the electrocatalytic oxidation reaction, the potential should be smaller than the threshold $\varepsilon_{0}$.

The net reaction rate is

$$
v^{n e t}=k_{\eta=0}^{f} \exp \left(\lambda^{f} \eta\right) C^{R}-k_{\eta=0}^{b} \exp \left(-\lambda^{b} \eta\right) C^{O}
$$

In equation (3), $\quad \eta=\varepsilon_{0}-\varepsilon$ is the overpotential. $k_{\eta=0}^{f}\left(k_{\eta=0}^{b}\right), C^{R}\left(C^{O}\right)$ and $\lambda^{f}\left(\lambda^{b}\right)$ are the rate constant at $\eta=0$, the reagent concentration of the forward (backward) reaction and the apparent barrier-reducing coefficient, respectively. For an oxidation potential $\eta_{i}>0, v^{\text {net }}$ is dominated by the forward reaction. And for $\eta_{i} \leq 0, v^{\text {net }}$ will be zero. The range of grids where the oxidation reaction can happen is truncated around NTs. Furthermore, The dielectric properties of the electrode and electrolyte is modeled in calculation of the potential $V$ near the electrode surface (5) where the charge 
density is computed using Gauss's law for electric field, $\rho=\varepsilon_{r} \varepsilon_{0} \nabla \cdot E$, in which $\varepsilon_{0}$ and $\varepsilon_{r}$ are the dielectric function for a vacuum and the materials (i.e., the electrode and the electrolyte), respectively. With the calculated potential, the dielectric properties of the electrode and electrolyte will affect the whole catalytic process both on the reaction and the kinetic.

\section{Details of numerical simulation.}

The kinetic equations are solved numerically with periodic boundary conditions on the $\mathrm{L} \times \mathrm{L}$ electrode surface, which is discretized to be a rectangle $\mathrm{N} \times \mathrm{N}$ lattice. $\mathrm{Pt}$ nanotubes (NTs) are distributed on the electrode surface in reality while what on the $\mathrm{L} \times \mathrm{L}$ electrode surface in simulations are discretized areas that are linear arranged. As the character lengths of the fields that are enhanced on those areas, $r_{0}$ remains accordant, potential distributions around NTs can be well fitted by those around linear arranged discretized areas.

The number of the NTs in our models is 20 and the length of them remains the same. Each of them arranges on the surface in the same direction with the same distance between each other. After selecting a spindle on each tube randomly and rotating the tubes around those spindles with angles randomly distributing in a certain range, the ordered arrangements of the tubes turn to be disordered. To illustrate the ordering of NTs, we set a parameter, $\beta$, which is the ratio between a range of the angle we choose to rotate the tubes and the max angle range, $90^{\circ}$. As $\beta$ increases gradually from 0 to 1 , the degree of order increases. Additionally, data of the models presented in our study are convinced enough via 30 times of ensemble averages. The reactions can be well reproduced with the parameter values list in Table $\mathrm{S} 3$ where $k_{B} T, \mathrm{~L}$ and $\mathrm{D}$ are considered to be basic units.

Similarly, simulations of MOR with nanoparticles (NPs) and nanoflakes (NFs) are based on the model of MOR with NTs. Since we consider the size of nanoparticles being similar to the size of the discretized areas consisting of NTs, parameters for NPs remain unchanged comparing to that for NTs (Table S4). A total number of 625 nanoparticles are placed on the surface. For ordered configuration, nanoparticles form 
a $25 \times 25$ lattice, while they lay on the surface of the electrode randomly as disordered configuration. As $\varepsilon=u_{0} r_{s}^{-0.7}$, where $r_{s}$ is determined by the size of the catalysts, parameters that are suitable for MOR with nanoflakes should be modified as follows (Table S5). Consisting of $2 \times 2$ sites, 100 nanoflakes lay on the electrode surface. Those flakes form a $10 \times 10$ lattice and separate on the surface averagely in ordered arrangement, while they are distributed randomly on the electrode as disordered configuration.

Deeper simulations into formic acid oxidation reaction (FAOR) could also be based on this model. Since the polarity of formic acid is considered to be higher than that of the methanol, $\varphi_{0}, C_{e q}$ and $k_{\eta=0}$ are increased based on the parameter values of MOR (Table S6).

\section{Details of the exposed avaliable surface area simulation.}

The available surface area of NTs is calculated by an ensemble average over more than 10000 configurations for each arrangement. Configurations are generated by the following procedure.

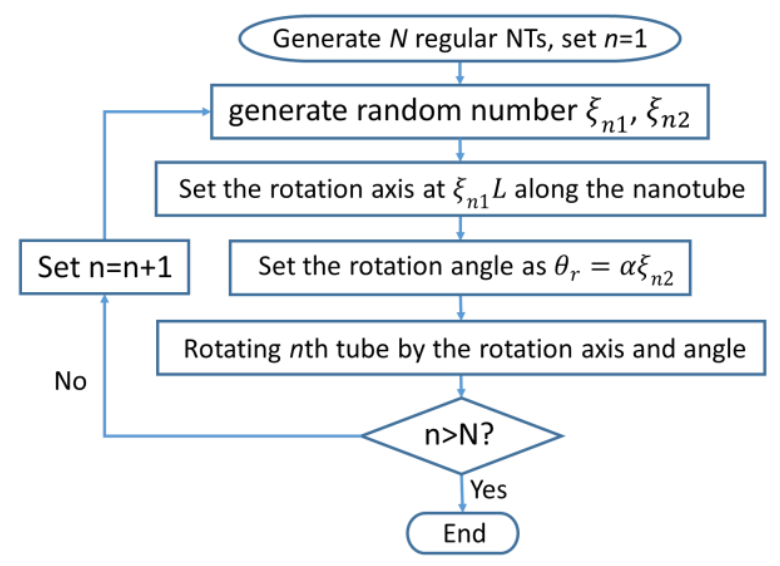

$>$ Step 1. Initially generate N NTs of length L regularly distributed with inter-NT distance $\mathrm{d}$, and set $n=1$.

$>$ Step 2. For the n-th NT, generate a random number $\xi_{n 1}$ according to the probability density function

$$
f\left(\xi_{n 1}\right)=1, \quad \xi_{n 1} \in[0,1]
$$


and chose a point locating at $\xi_{n 1} L$ along the NT as the rotation axis.

$>$ Step 3. Generate a random number $\xi_{n 2}$ according to the probability density function

$$
f\left(\xi_{n 2}\right)=\frac{1}{2}, \quad \xi_{n 2} \in[-1,1]
$$

and set $\theta_{r}=\alpha \xi_{n 2}$ as the rotation angle.

Step 4. Rotating the NT over the rotation angle $\theta_{r}$ according to the rotation axis $\xi_{n 1} L$.

Step 5. Set $n=n+1$, and repeat Step 2-4 till all NTs has been operated.

Mathematically, the above procedure provides an operator $\hat{p}$ for any point on the NT. Locations before and after the operation ((xn, yn) and (xn', yn'), respectively) can be related by

$$
\left(\begin{array}{l}
x_{n}{ }^{\prime} \\
y_{n}{ }^{\prime}
\end{array}\right)=\hat{p} \cdot\left(\begin{array}{l}
x_{n} \\
y_{n}
\end{array}\right)=\left(\begin{array}{cc}
\cos \theta_{r} & -\sin \theta_{r} \\
\sin \theta_{r} & \cos \theta_{r}
\end{array}\right)\left(\begin{array}{c}
x_{n}-\xi_{n 1} L \\
y_{n}-n d
\end{array}\right)+\left(\begin{array}{c}
\xi_{n 1} L \\
n d
\end{array}\right) .
$$

Besides, the randomness of the arrangement can be controlled by the parameter $\alpha$. As $\alpha$ increases from $0^{\circ}$ to $90^{\circ}$, the arrangement of NTs will change from the regular one to be completely random. Thus the order parameter

$$
\beta=1-\frac{\alpha}{90^{\circ}}
$$

is defined to measure the ordering directly.

With the generated configurations, the available surface area $S_{a v a}$ of NTs is calculated as follows. By discretizing the tubes into $M$ small segments whose available active area is $s_{0}$, the covered area of tubes for a given configuration can be conveniently obtained by counting $m_{\text {olp }}$ the number of overlapped segment pairs as follows, 


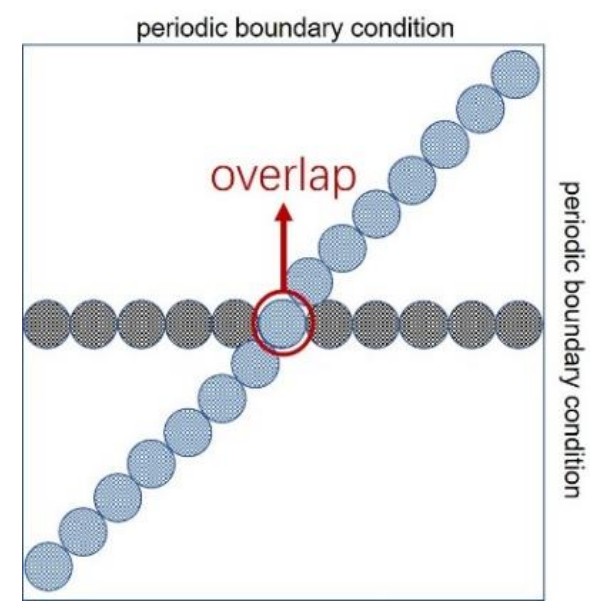

and thus

$$
S_{a v a}=\left\langle s_{0}\left(M-m_{o l p}\right)\right\rangle,
$$

where $\langle\cdot\rangle$ means the ensemble average over 10000 configurations.

4. Determination of orientational parameter. Image-J software with the Orientation J plug-in was employed to quantify the ordering, i.e., the orientational parameter $\mathrm{S}$ of the TEM images of 1D Pt-based assemblies. The Orientation J distribution analysis program was set to a Gaussian gradient over 10 pixels. The result of the analysis was a distribution of space-correlation alignment intensity $(\mathrm{N})$ as a function of angle $(\delta)$. The horizontal direction with a maximum correlation intensity was set as the main director in all TEM images. Then $\mathrm{S}$ was obtained by averaging over the angle distribution:

$$
\mathrm{S}=\frac{\sum N\left[2(\cos \delta)^{2}-1\right]}{\sum N}
$$

The determined $\mathrm{S}$ can change from 0 (completely disordered) to 1 (perfectly ordered). 


\section{Supplementary Figures}

(a)

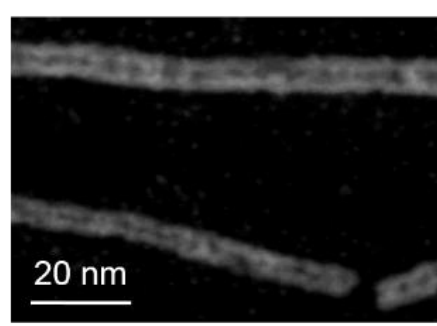

(d)

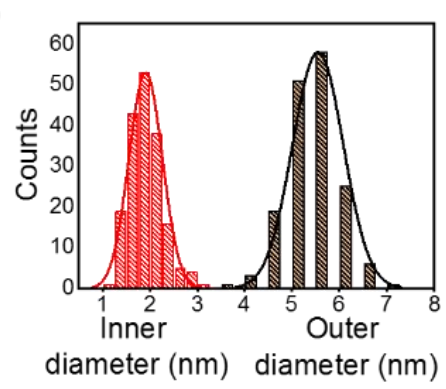

(b)

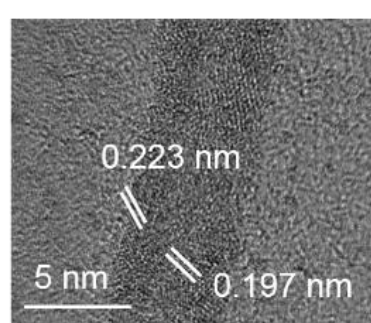

(e) 40

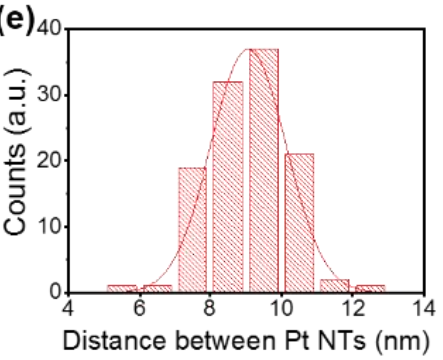

(c)

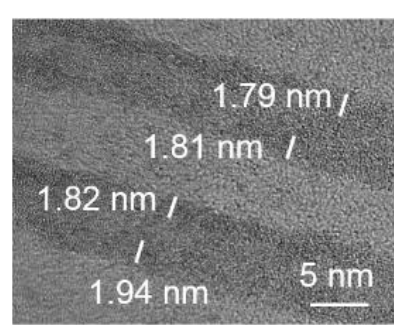

(f)

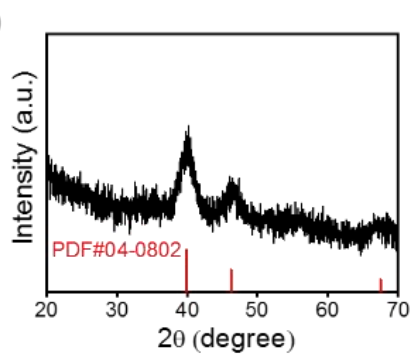

Figure S1. Morphological and structural characterizations of the Pt NTs. (a) High-angle annular dark field image-scanning transmission electron microscope (HAADF-STEM) image shows the hollow tubular structure of the Pt NTs. (b) High-resolution transmission electron microscopy (HR-TEM) image shows the lattice spacings of 0.223 $\mathrm{nm}$ and $0.197 \mathrm{~nm}$, corresponding to the lattice planes of (111) and (220), respectively. (c) HR-TEM image shows the Pt NTs have a wall thickness of $1.84 \pm 0.07 \mathrm{~nm}$. (d) Size distributions of Pt NTs show that the obtained Pt NTs have an outer and inner diameter of $5.55 \pm 0.54 \mathrm{~nm}$ and $1.90 \pm 0.35 \mathrm{~nm}$, respectively. (e) Size distribution of the distance between Pt NTs shows the interval between two Pt NT is $9.07 \pm 1.06 \mathrm{~nm}$. (f) RD pattern of Pt NTs. All peaks can be indexed to cubic Pt, which is consistent with the standard literature (JCPDS No.04-0802).
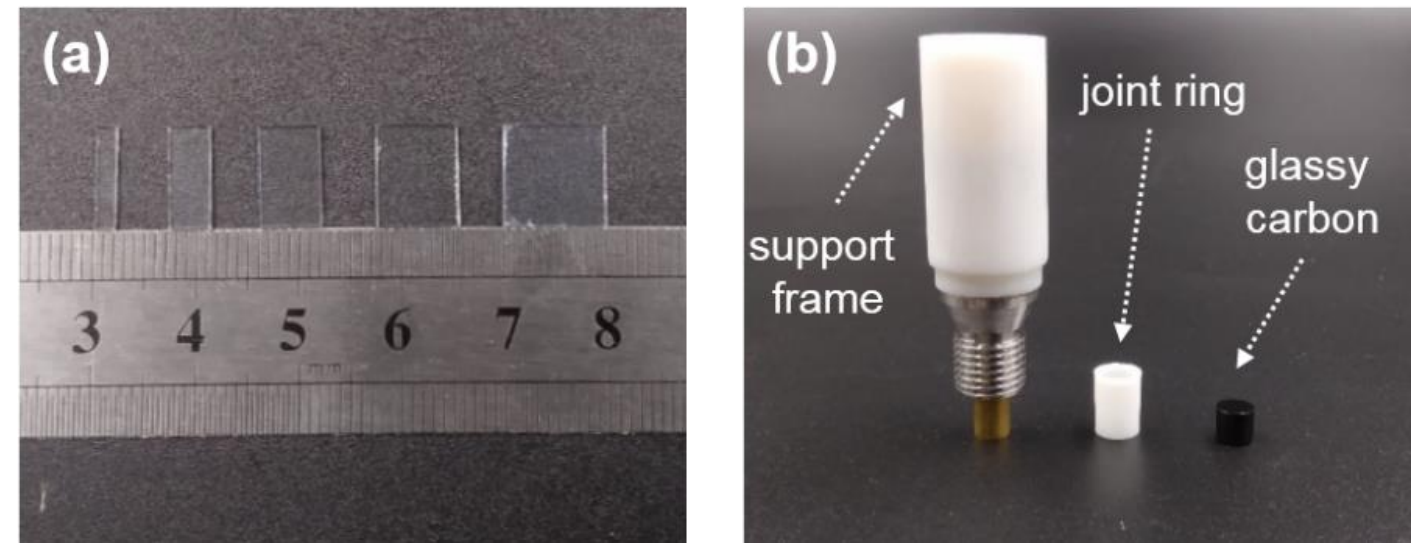

Figure S2. Optical photograph of glass substrates with various areas and detachable glassy carbon electrode. (a) Glass substrates with width of $0.2,0.4,0.6,0.8$ and $1.0 \mathrm{~cm}$, length of $1.0 \mathrm{~cm}$. (b) Front view of support frame, joint ring and glassy carbon of the electrode. 
(a)

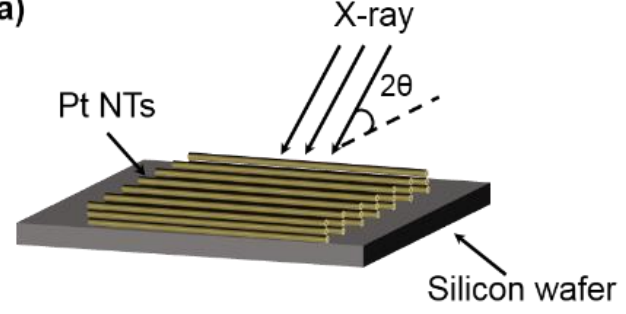

(b)

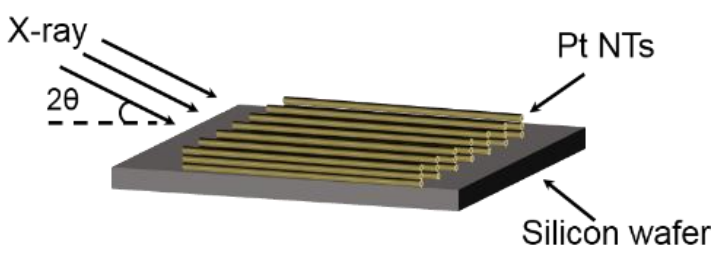

Figure S3. Schematic illustrations and result of the small angle X-ray diffraction (SAXRD) measurement. Schematic illustrations show that the incident direction of Xray can be perpendicular (a) and parallel (b) to the array direction. Three paralleled $\mathrm{Pt}$ NTs array layers were transferred on $2 \mathrm{~cm} \times 2 \mathrm{~cm}$-sized silicon wafer. The incident Xray scan angle $(2 \theta)$ ranges from 0.5 to 5 degree.

(a)

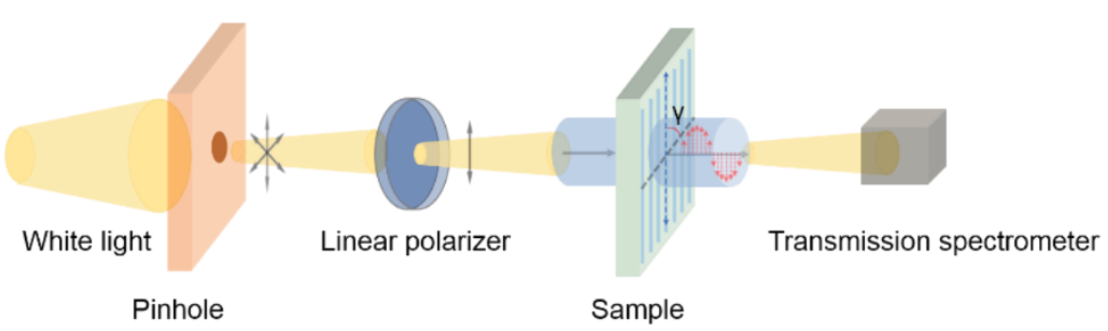

(b)

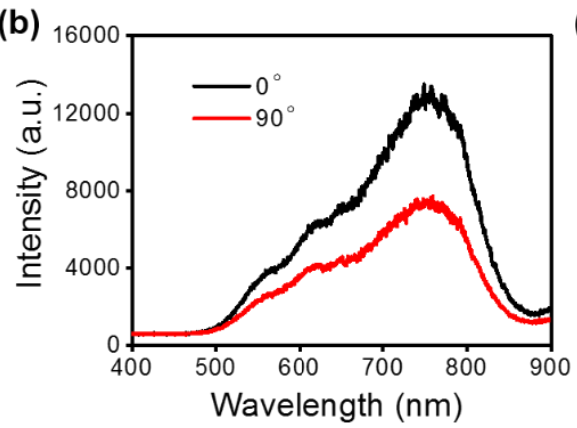

(c)

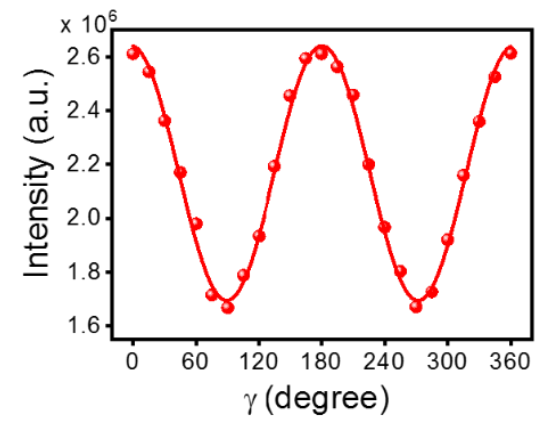

Figure S4. Polarized light transmission spectrum tests. (a) Schematic illustration shows the experimental setup for polarized light transmission spectrum test. A linear polarizer was used to produce polarized white light. (b) The spectrum shows the maximum and minimum intensity of transmitted light when the sample stage is parallel $\left(\gamma=0^{\circ}\right)$ and perpendicular $\left(\gamma=90^{\circ}\right)$ to the incident light, respectively. (c) The polarization dependence of transmission intensity on the rotation angle $(\gamma)$ of sample stage shows a sine function. Dot: experimental data; line: fitted curve. 

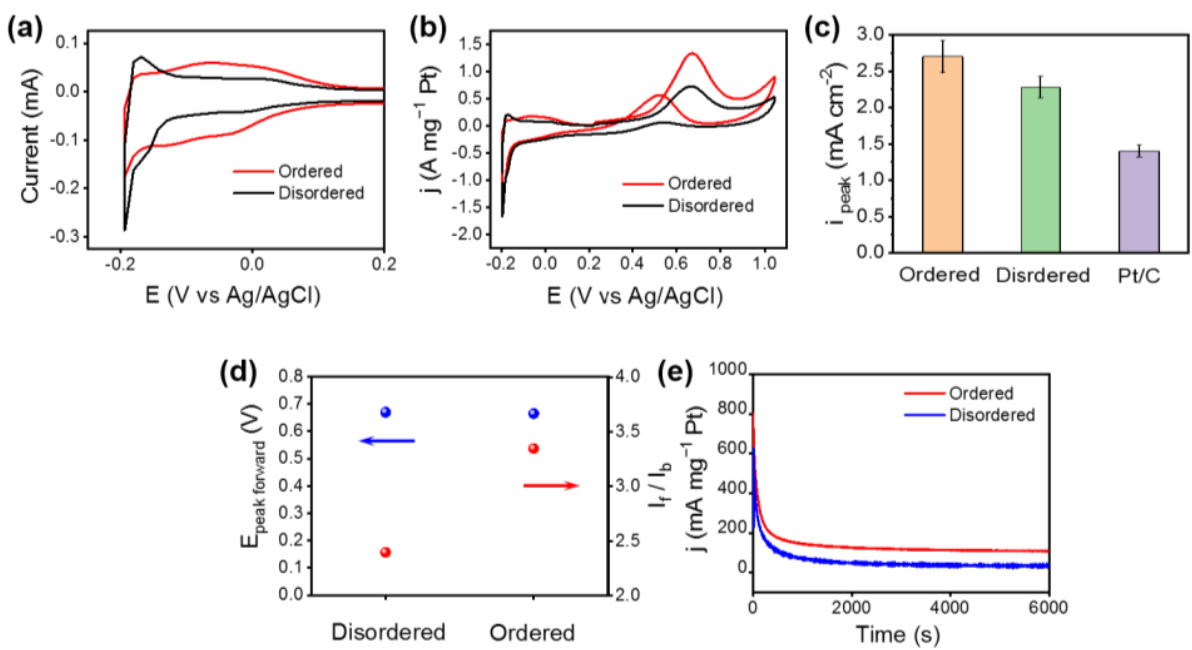

Figure S5. Methanol oxidation reaction (MOR) measurements of ordered and disordered Pt NTs nanostructures. (a) The partial enlarged detail from $-0.2 \mathrm{~V}$ to $0.2 \mathrm{~V}$ in cyclic voltammetry (CV) curves in $0.1 \mathrm{M} \mathrm{HClO}_{4}$ at a scan rate of $250 \mathrm{mV} \mathrm{s}^{-1}$ show the ordered Pt NTs monolayer have a more typical underpotentially deposited hydrogen adsorption/desorption charge than disordered counterpart. (b) MOR performances show ordered Pt NTs monolayer has a better catalytic activity for MOR. The measurements were carried out in $0.1 \mathrm{M} \mathrm{HClO}_{4}+1.0 \mathrm{M} \mathrm{CH}_{3} \mathrm{OH}$ after 50 cycles of $\mathrm{CV}$ activation at a scan rate of $50 \mathrm{mV} \mathrm{s}^{-1}$. (c) Histograms of area activities of Pt NT monolayer for MOR in $0.1 \mathrm{M} \mathrm{HClO}_{4}+1.0 \mathrm{M} \mathrm{CH}_{3} \mathrm{OH}$ at a sweep rate of $50 \mathrm{mV} \mathrm{s}^{-1}$, which are standardized by active surface areas (ECSA). Error bars correspond to the standard deviations taken over at least three measurements. (d) $E_{\text {peak forward }}$ and $I_{f} / I_{b}$ comparisons show that ordered Pt NTs monolayer exhibits close peak position to disordered Pt NTs monolayer, and the $\mathrm{I}_{\mathrm{f}} / \mathrm{I}_{\mathrm{b}}$ for ordered Pt NTs monolayer was much larger (3.35) than that of disordered Pt NTs monolayer (2.39). (e) Chronoamperograms for the MOR at applied potential of $0.55 \mathrm{~V}$ vs. $\mathrm{Ag} / \mathrm{AgCl}$ of ordered and disordered $\mathrm{Pt}$ NTs monolayers in $0.1 \mathrm{M} \mathrm{HClO}_{4}+1.0 \mathrm{M} \mathrm{CH}_{3} \mathrm{OH}$ a scan rate of $50 \mathrm{mV} \mathrm{s}^{-1}$.
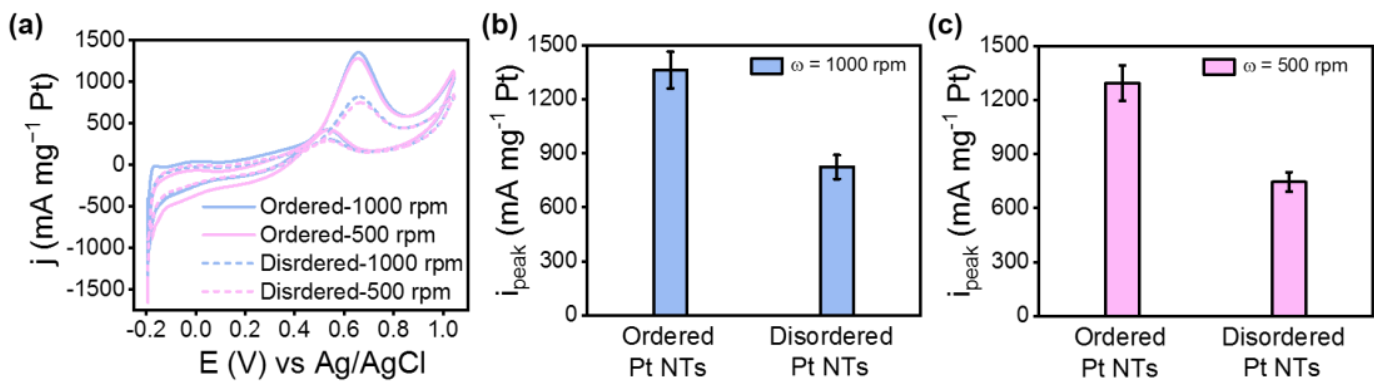

Figure S6. MOR performances of Pt NTs model catalyst recorded with different rotating speeds applied. (a) MOR performances of ordered and disordered Pt NT catalyst recorded under different rotating speeds of 500 and $1000 \mathrm{rpm}$. (b, c) Histograms

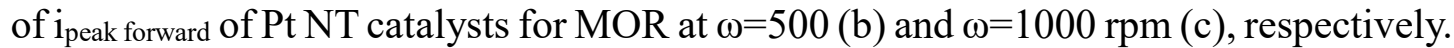
Error bars correspond to the standard deviations taken over at least three measurements. 
(a)

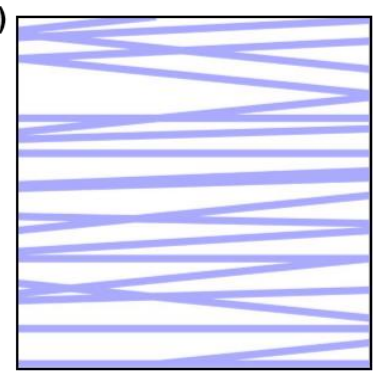

(b)

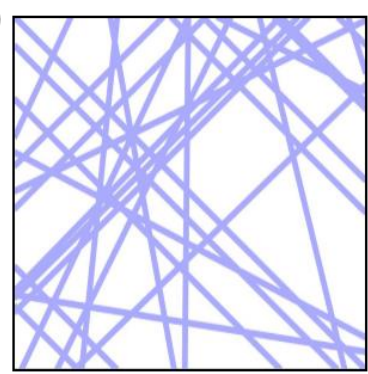

(c)

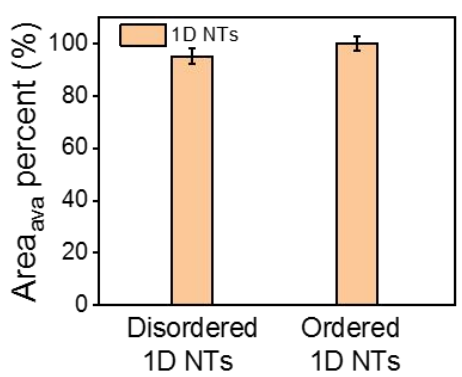

Figure S7. Statistical analysis of the exposed available surface areas in different patterns. (a) Ordered NP pattern. (b) Disordered NP pattern. (c) Histogram of the area available percents in different patterns.
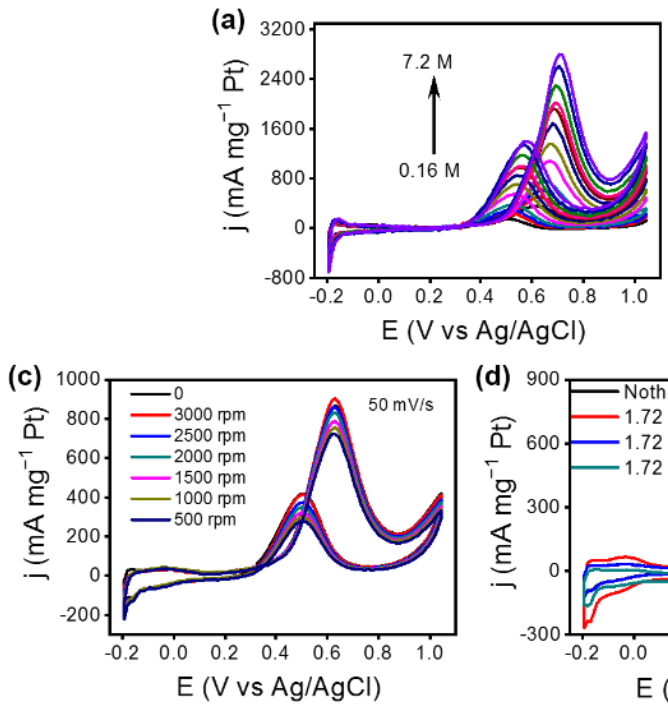
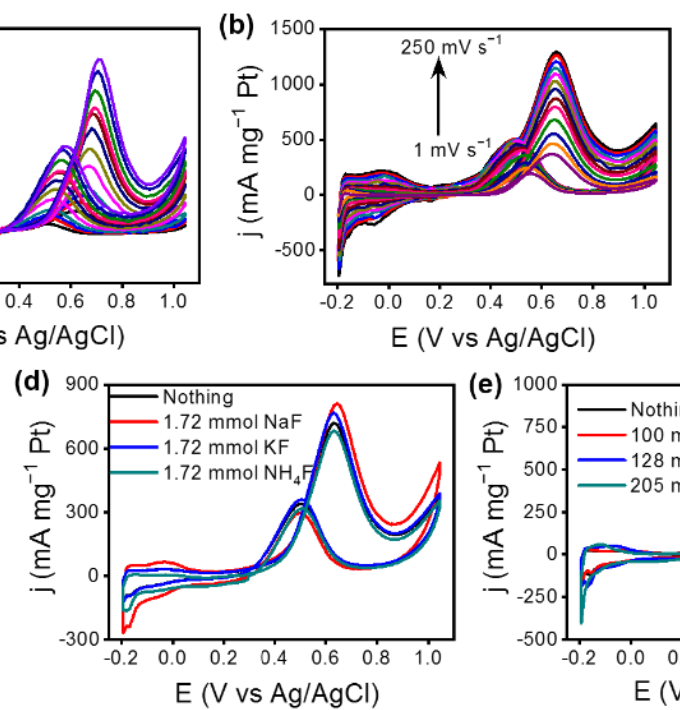

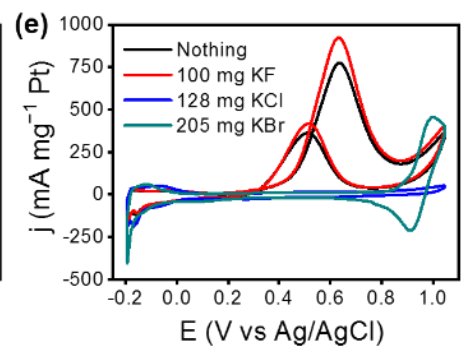

Figure S8. MOR performances of Pt NTs model catalyst recorded with different electrochemical parameters. (a) Concentrations of $\mathrm{CH}_{3} \mathrm{OH}$ from 0.16 to $8.8 \mathrm{M}$, (b) scan rates from 1 to $250 \mathrm{mV} \mathrm{s}^{-1}$ and (c) rotating speed from 0 to $3000 \mathrm{rpm}$. (d-e) MOR performances were recorded in $0.1 \mathrm{M} \mathrm{HClO}_{4}+1.0 \mathrm{M} \mathrm{CH}_{3} \mathrm{OH}$ with adding different electrolytes at a sweep rate of $50 \mathrm{mV} \mathrm{s}^{-1}$. Adding $\mathrm{F}^{-}$can elevate the current density of MOR due to the prominent electrostatic attraction between $\mathrm{F}^{-}$and $\mathrm{CH}_{3} \mathrm{OH}$ molecule. Adding $\mathrm{Cl}^{-}$and $\mathrm{Br}^{-}$inhibits the MOR and even motivates HER, respectively, resulting from the competition between $\mathrm{K}^{+}$and the anions. 

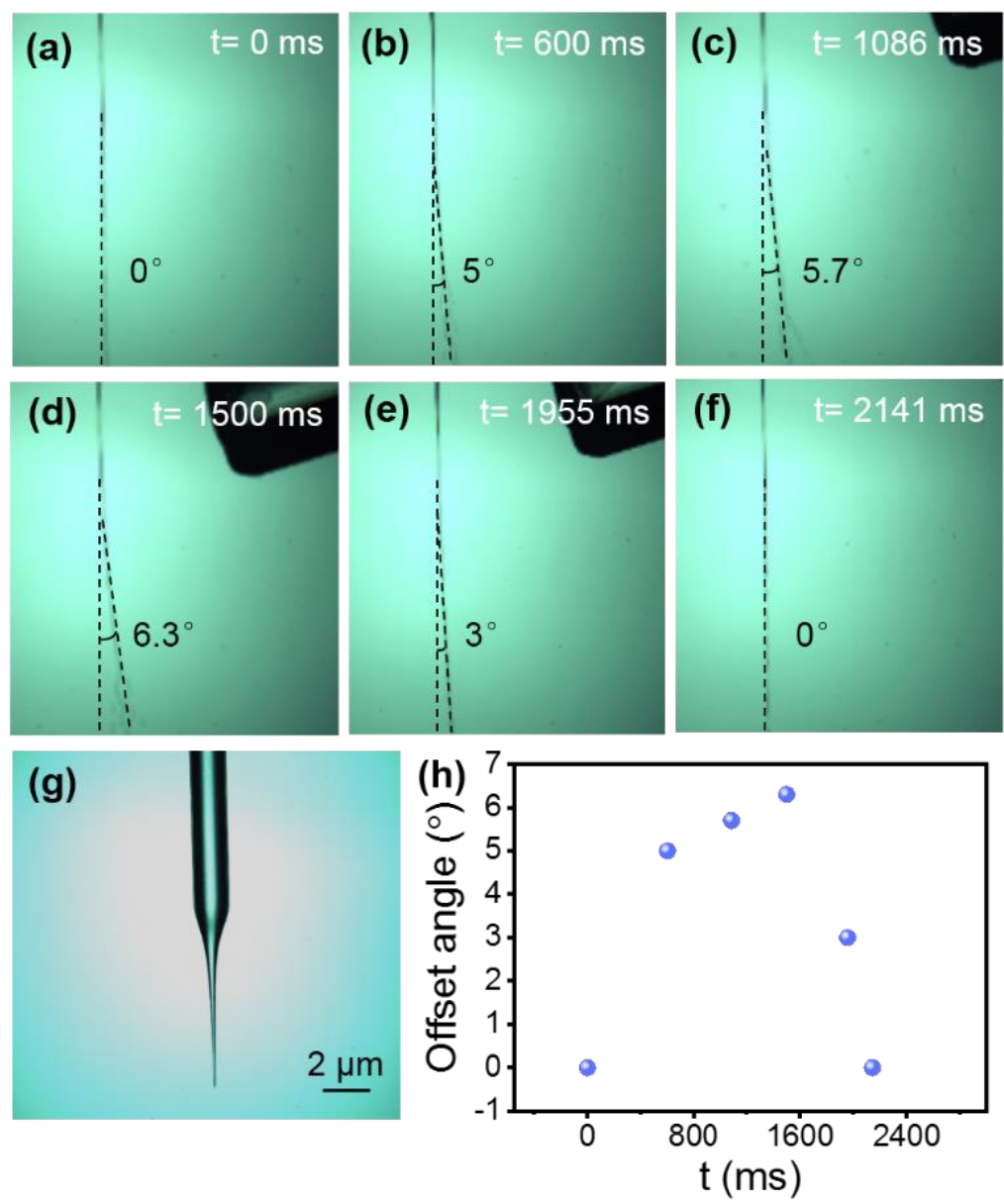

Figure S9. Movement of $\mathrm{CH}_{3} \mathrm{OH}$ jet in electrostatic field. Optical photograph of $\mathrm{CH}_{3} \mathrm{OH}$ jet in electrostatic field captured by the high-speed camera at time of (a) $\mathrm{t}=0$ $\mathrm{ms}$, (b) $600 \mathrm{~ms}$, (c) $1086 \mathrm{~ms}$, (d) $1500 \mathrm{~ms}$, (e) $1955 \mathrm{~ms}$ and (f) $2141 \mathrm{~ms}$. (g) Optical photograph of the needle tubing to produce $\mathrm{CH}_{3} \mathrm{OH}$ jet. The diameter at the tip is $76.7 \mu \mathrm{m}$. The efflux velocity of methanol jet controlled by syringe pump is $300 \mu \mathrm{L}$ $\mathrm{min}^{-1}$. The rate of methanol jet at the tip is $1.08 \mathrm{~mm} \mathrm{~s}^{-1}$. (h) Deviation of $\mathrm{CH}_{3} \mathrm{OH}$ jet induced by electrostatic field. At time of $600 \mathrm{~ms}$, glass rod with static electricity approached the $\mathrm{CH}_{3} \mathrm{OH}$ jet with a jet velocity of $1.08 \mathrm{~mm} \mathrm{~s}^{-1}$. Upon the approaching, the $\mathrm{CH}_{3} \mathrm{OH}$ jet deviated from its free fall orbit, where the closer the glass rod got, the larger the offset angle was. After withdrawing the electrostatic field, $\mathrm{CH}_{3} \mathrm{OH}$ jet returned immediately to its previous route from the maximum angle of appr. $6.5^{\circ}$ within $1 \mathrm{~s}$. 


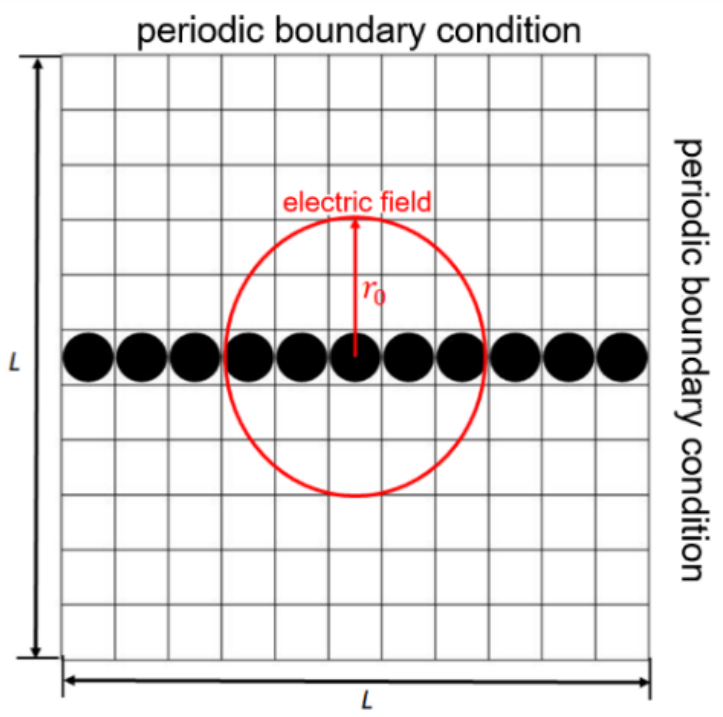

Figure S10. The numerical simulation setup. Black balls denote the Pt NTs and the L $\times$ L surface is discretized into $\mathrm{N} \times \mathrm{N}$ lattice with periodic boundary conditions.
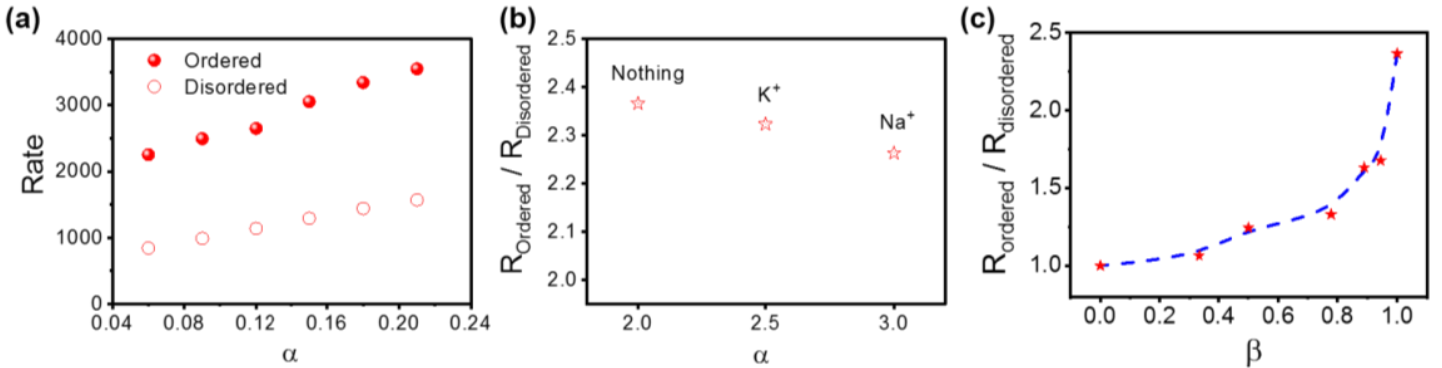

Figure S11. Kinetic simulation results of Pt NTs for MOR. (a) The apparent rate for MOR on ordered (solid circle) and disordered (hollow circle) Pt NTs with various interaction strength $\alpha$. (b) The ratio between the rate of ordered NTs and that of disordered NTs in different electrolyte solutions with different values of $\alpha$. (c) The ratio between the reactions of ordered and disordered NTs with the variation of the degree of order, $\beta$. 
(a)

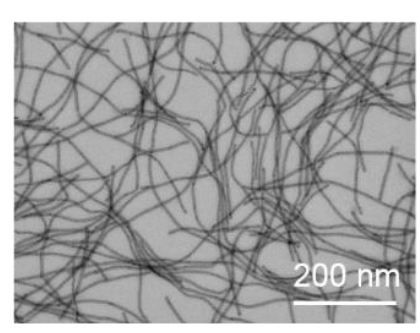

(c)

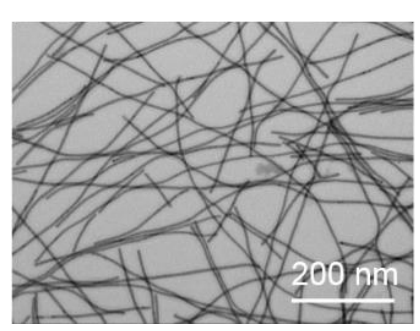

(b)

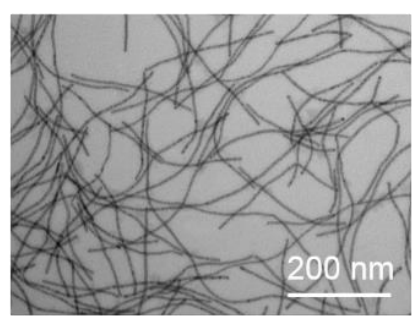

(d)

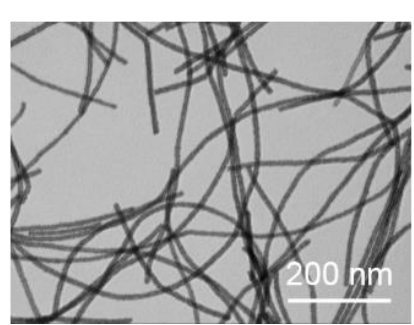

Figure S12. Representative TEM images of other one-dimensional (1D) Pt-based multicomponent nanostructures. (a) PtTe NWs, (b) PtPdTe NWs, (c) PtAuTe NWs, (d) PtPdRu NTs.
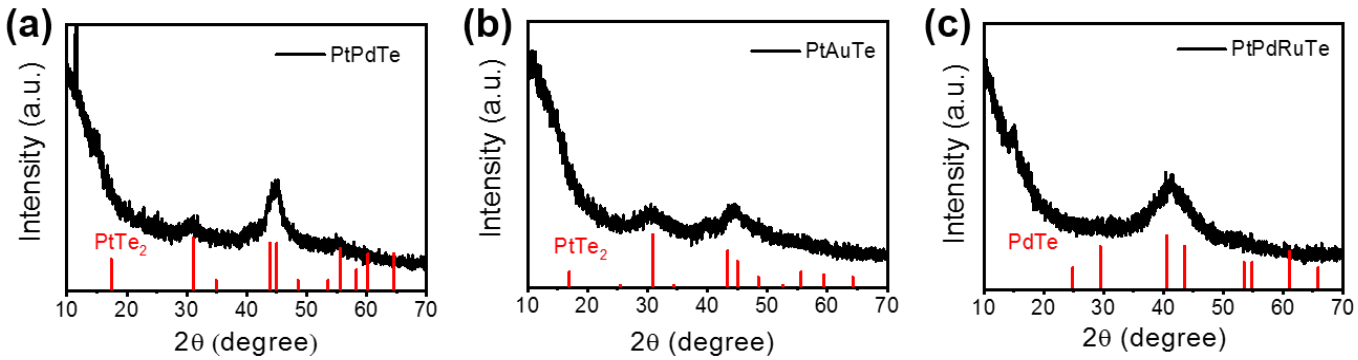

Figure S13. X-ray diffraction (XRD) pattens of 1D Pt-based multicomponent nanostructures. (a) PtPdTe NWs, (b) PtAuTe NWs, (c) PtPdRuTe NTs.

(a)

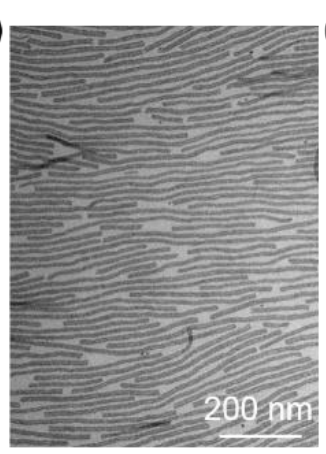

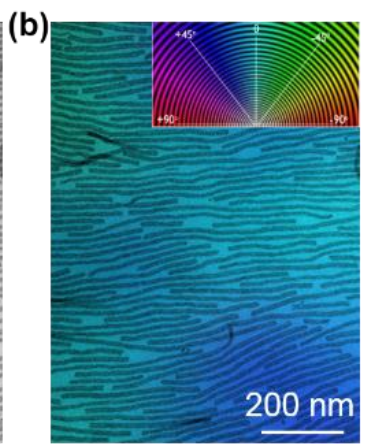

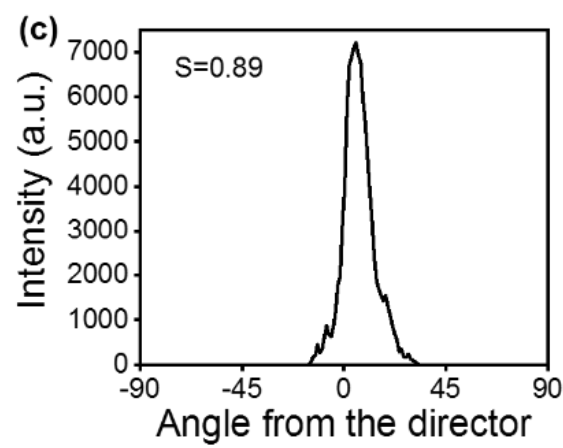

Figure S14. Ordering determination of ordered PtTe monolayer. (a) TEM image of PtTe NWs monolayer. (b) The angular space correlation alignment intensity distributions. Inset: circular color map coding. (c) The resulting orientational parameter $\mathrm{S}$. $\mathrm{S}=0.89$. 

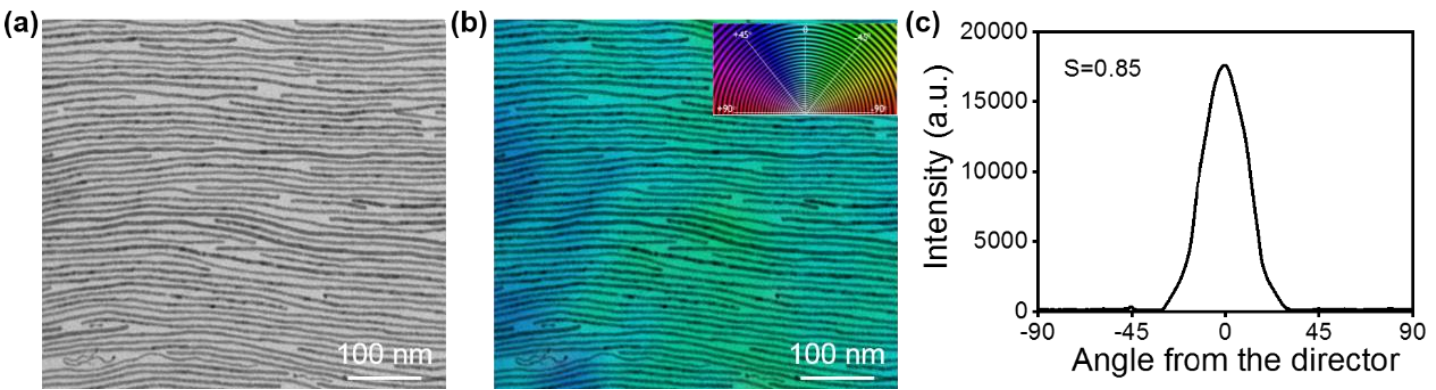

Figure S15. Ordering determination of ordered PtPdTe monolayer. (a) TEM image of PtPdTe NWs monolayer. (b) The angular space correlation alignment intensity distributions. Inset: circular color map coding. (c) The resulting orientational parameter S. $S=0.85$.

(a)

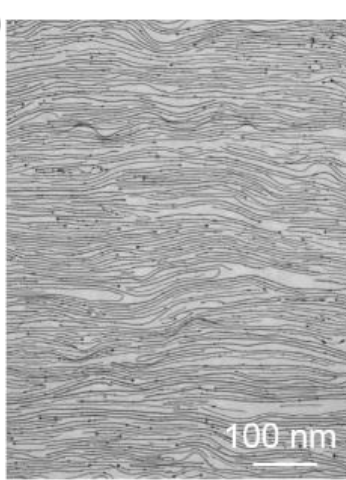

(b)

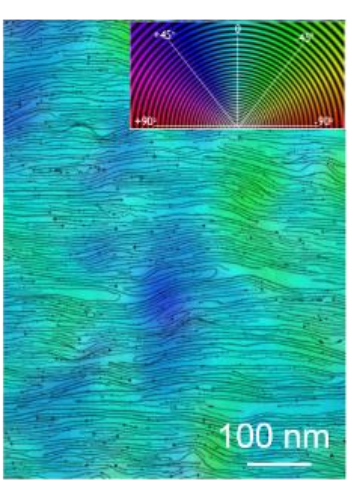

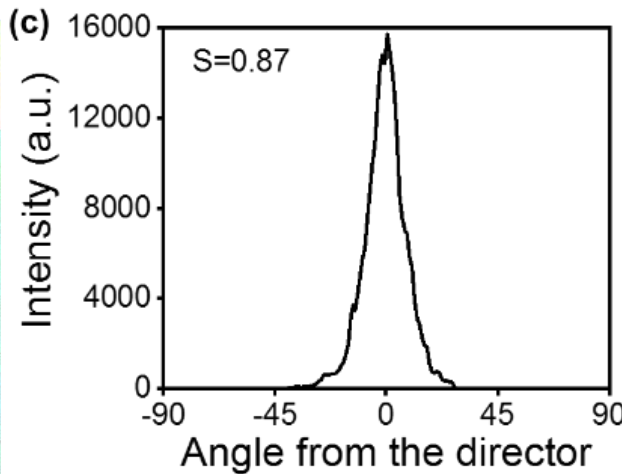

Figure S16. Ordering determination of ordered PtAuTe monolayer. (a) TEM image of PtAuTe NWs monolayer. (b) The angular space correlation alignment intensity distributions. Inset: circular color map coding. (c) The resulting orientational parameter S. $S=0.87$.
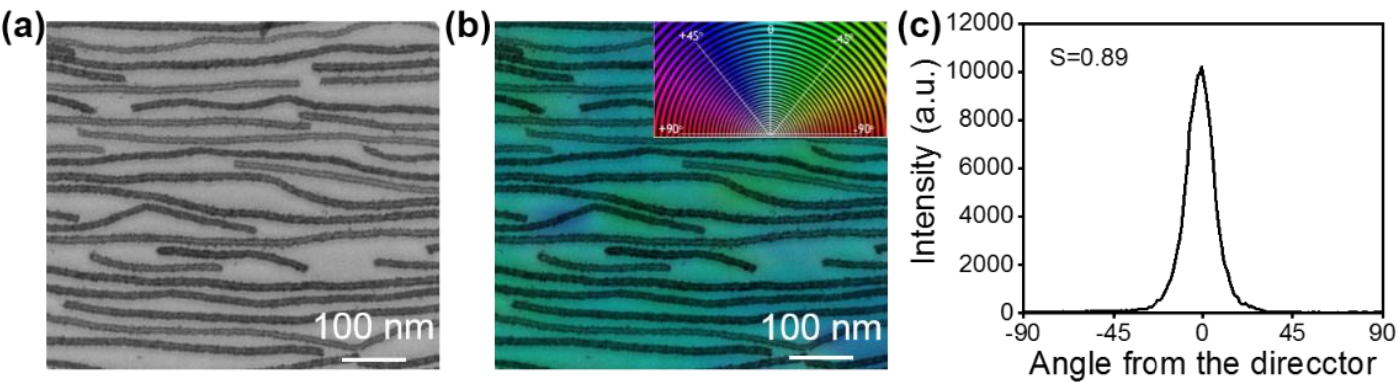

Figure S17. Ordering determination of ordered PtPdRuTe monolayer. (a) TEM image of PtAuTe NWs monolayer. (b) The angular space correlation alignment intensity distributions. Inset: circular color map coding. (c) The resulting orientational parameter S. $S=0.89$. 

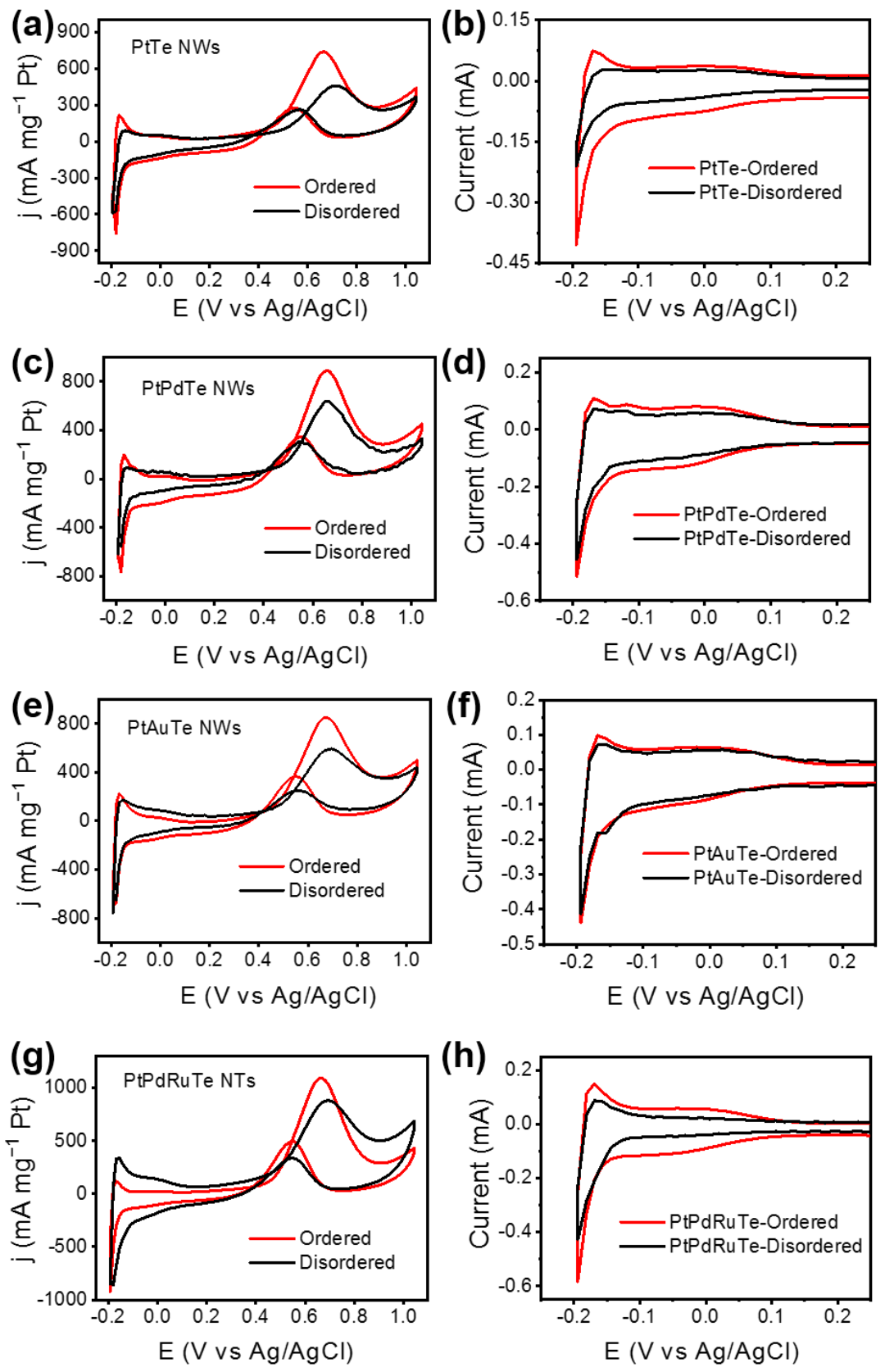

Figure S18. MOR performances of other 1D Pt-based multicomponent nanostructures. CV profiles of ordered and disordered (a) PtTe NWs, (c) PtPdTe NWs, (e) PtAuTe NWs and (g) PtPdRuTe NTs. The partial enlarged detail of CV curves of ordered and disordered (b) PtTe NWs, (d) PtPdTe NWs, (f) PtAuTe NWs and (h) PtPdRuTe NTs. The results show high order introduced into these multicomponent $1 \mathrm{D}$ systems exerts general advantages in MOR performance and active areas exposed to the electrolyte. MOR measurements were performed in $0.1 \mathrm{M} \mathrm{HClO}_{4}+1.0 \mathrm{M} \mathrm{CH}_{3} \mathrm{OH}$ at a sweep rate of $50 \mathrm{mV} \mathrm{s}^{-1}$. 
(a)

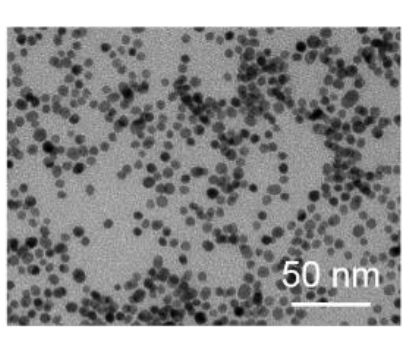

(b)

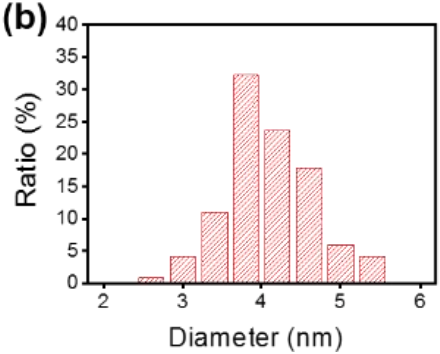

(c)

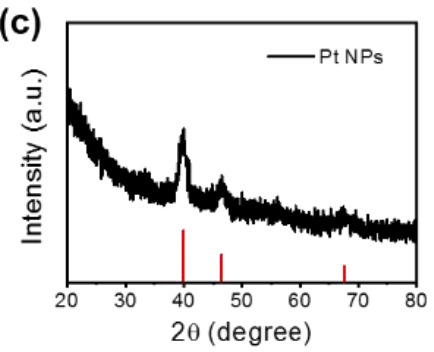

Figure S19. Morphological and structural characterizations of Pt-based NPs. (a) Representative TEM images of Pt NPs. (b) Size distribution of Pt NPs. The histograms show the diameter of Pt NPs is $3.9 \pm 0.34 \mathrm{~nm}$. (c) XRD pattern of Pt NPs.
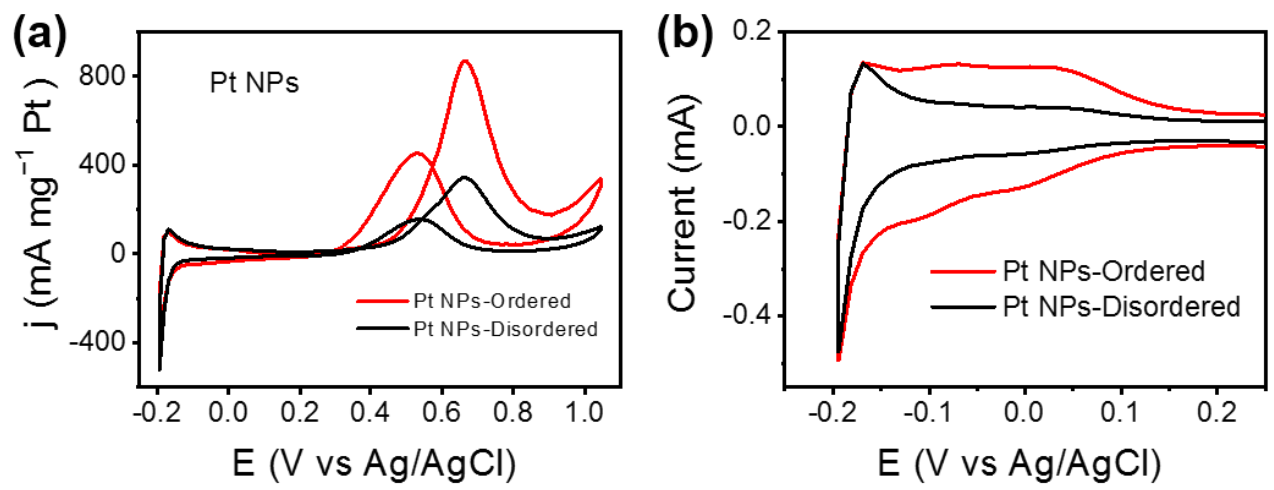

Figure S20. MOR performances of Pt-based NPs. (a) MOR profiles of ordered and disordered Pt NPs. (b) Partial enlarged detail of CV curves of ordered and disordered Pt NPs. The results show high order introduced into these multicomponent 0D systems exerts general advantages in MOR performance and active areas exposed to the electrolyte. MOR measurements were performed in $0.1 \mathrm{M} \mathrm{HClO}_{4}+1.0 \mathrm{M} \mathrm{CH}_{3} \mathrm{OH}$ at a sweep rate of $50 \mathrm{mV} \mathrm{s}^{-1}$.

(a)

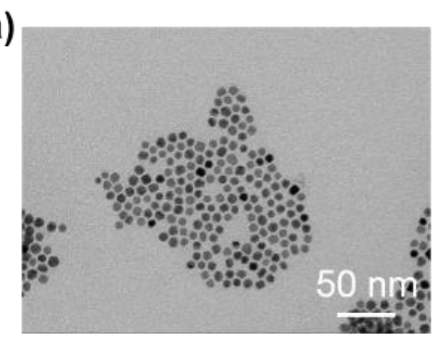

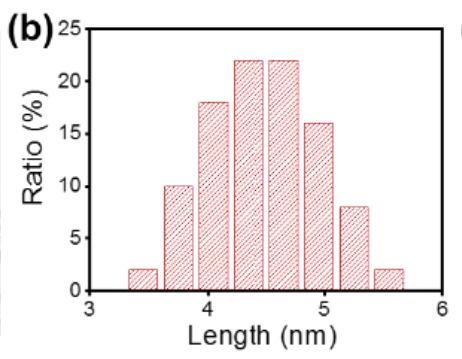

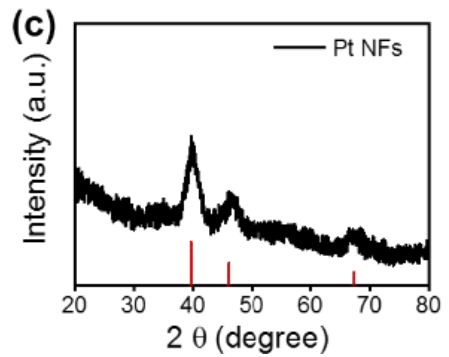

Figure S21. Morphological and structural characterizations of Pt-based NFs. (a) Representative TEM images of Pt NFs. (b) Size distribution of Pt. The histograms show the diameter of Pt NFs is $4.55 \pm 0.30 \mathrm{~nm}$. (c) XRD pattern of Pt NFs. 

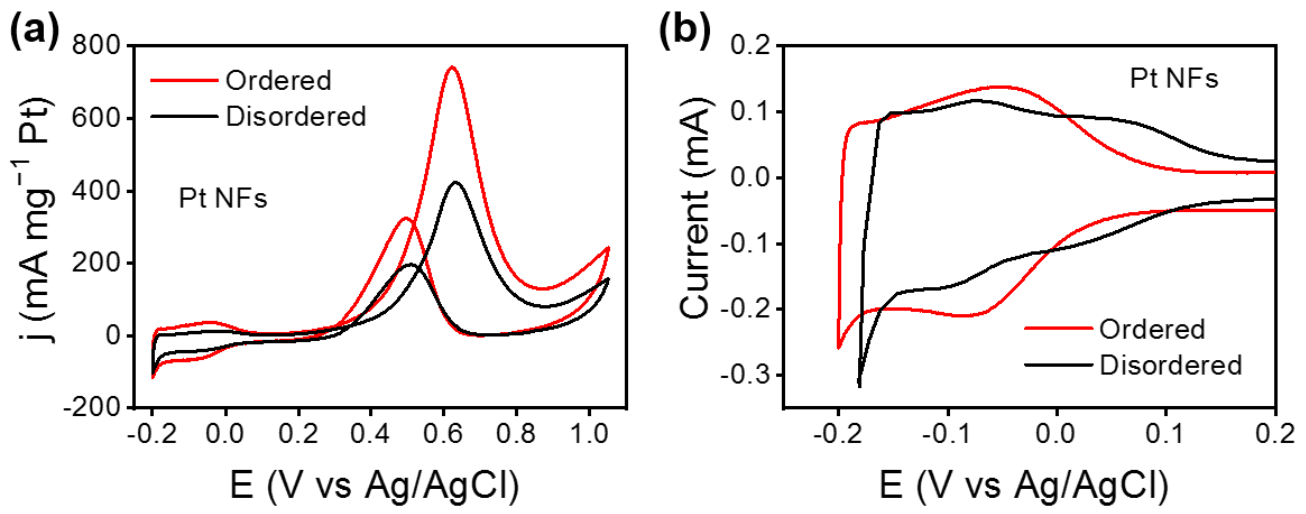

Figure S22. MOR performances of Pt-based NFs. (a) MOR profiles of ordered and disordered Pt NFs. (b) Partial enlarged detail of CV curves of ordered and disordered Pt NFs. MOR measurements were performed in $0.1 \mathrm{M} \mathrm{HClO}_{4}+1.0 \mathrm{M} \mathrm{CH}_{3} \mathrm{OH}$ at a sweep rate of $50 \mathrm{mV} \mathrm{s}^{-1}$.

(a)
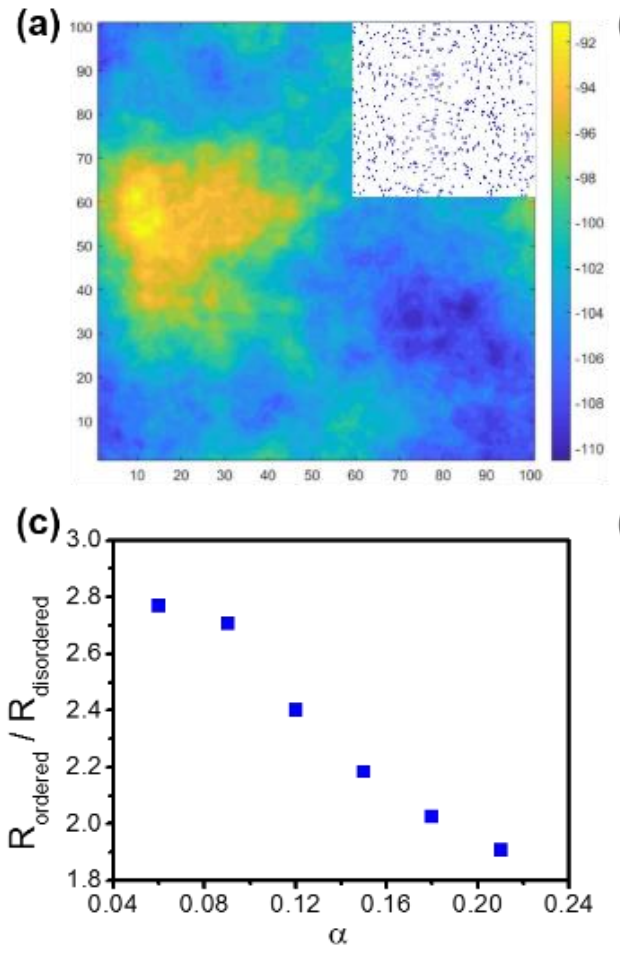

(b)

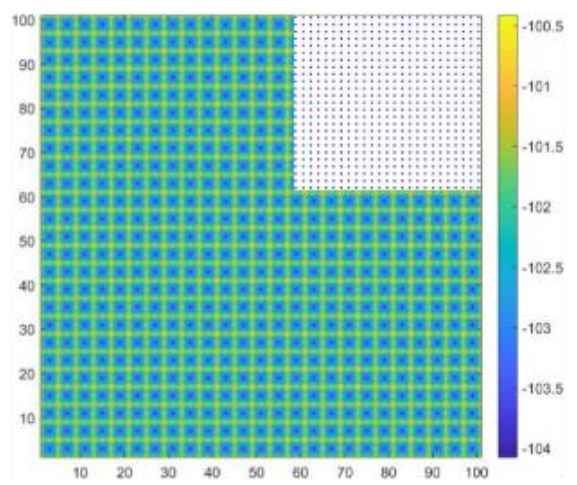

(d)

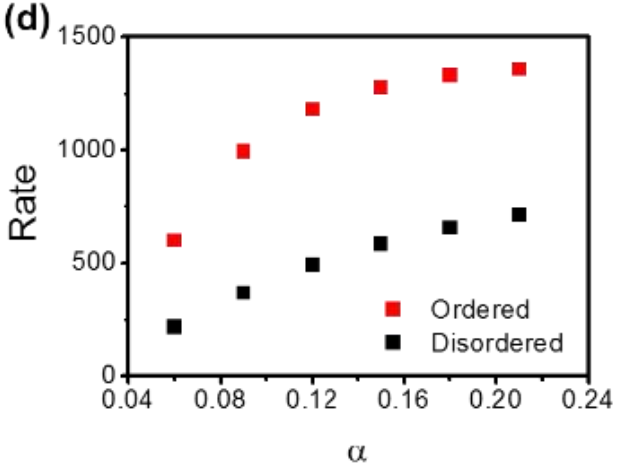

Figure S23. Kinetic simulation results of Pt NPs for MOR. (a) Potential of the field enhanced by ordered arranged Pt NPs. (b) Potential of the field enhanced by disordered arranged Pt NPs. (c) The apparent rate for the reaction of MOR on ordered (red square) and disordered (black square) Pt NPs with various $\alpha$. (d) The ratio between the rate of ordered NPs and that of disordered NPs with the different values of $\alpha$. 
(a)
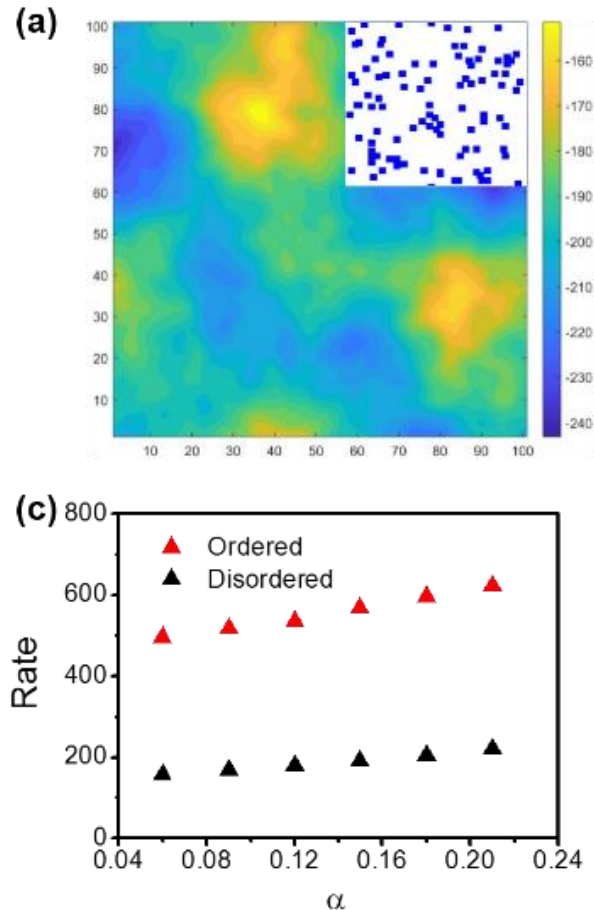

(b)
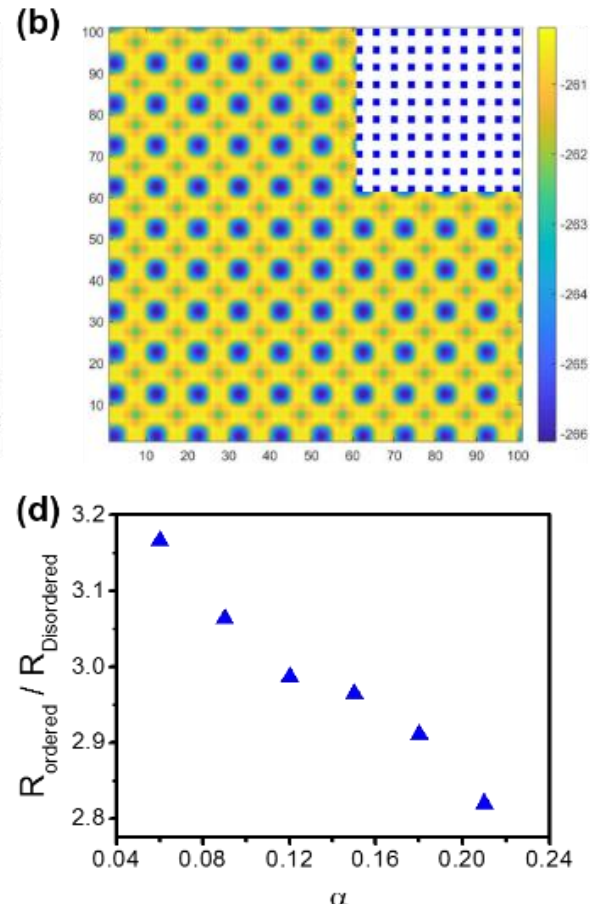

Figure S24. Kinetic simulation results of Pt NFs for MOR. (a) Potential of the field enhanced by ordered arranged Pt NFs. (b) Potential of the field enhanced by disordered arranged Pt NFs. (c) The apparent rate for the reaction of MOR on ordered (red triangle) and disordered (black triangle) Pt NFs with various $\alpha$. (d) The ratio between the rate of ordered NFs and that of disordered NFs with the different values of $\alpha$.

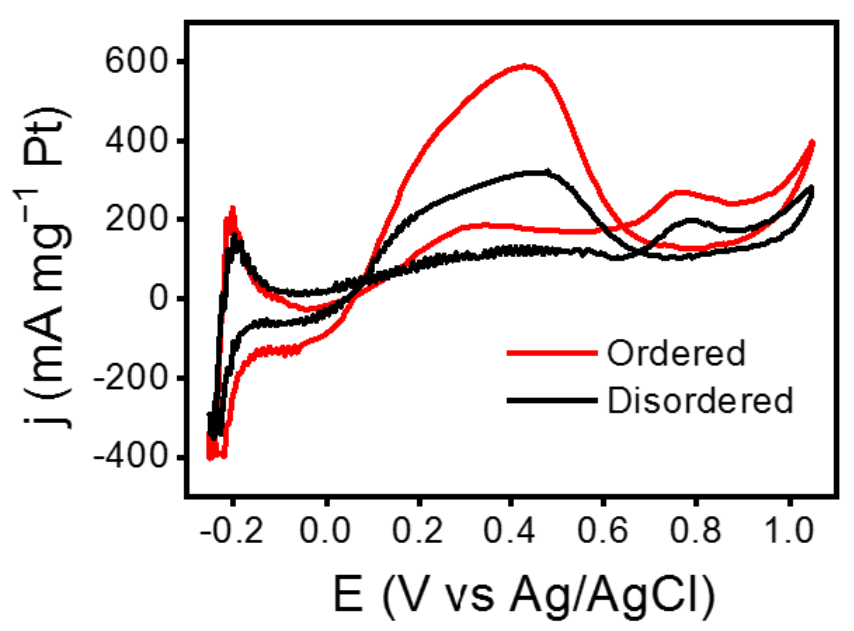

Figure S25. Formic acid oxidation reaction (FAOR) measurements of ordered and disordered Pt NTs monolayer. MOR performances were obtained in $0.1 \mathrm{M} \mathrm{HClO}_{4}+1.0$ $\mathrm{M} \mathrm{HCOOH}$ after 50 cycles of CV activation at a scan rate of $50 \mathrm{mV} \mathrm{s}^{-1}$. 

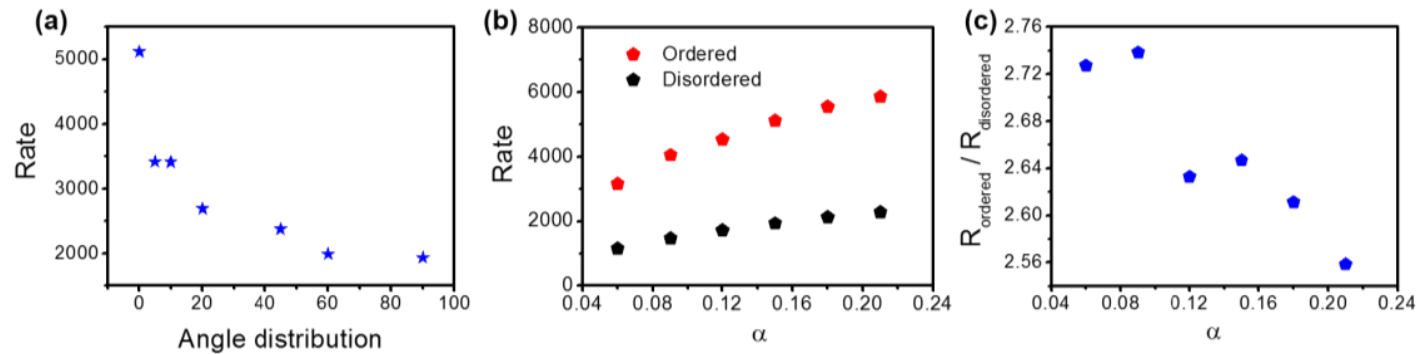

Figure S26. Kinetic simulation results of Pt NTs for FAOR. (a) The apparent rate for FAOR on ordered Pt NTs with various ordering degrees. (b) The apparent rate for FAOR on ordered (red pentagon) and disordered (black pentagon) Pt NTs with various $\alpha$. (c) The ratio between the rate of ordered NTs and that of disordered NTs with different values of $\alpha$.

\section{Supplementary Tables}

Table S1. Component contents in Pt-based nanostructures.

\begin{tabular}{llllll}
\hline \multicolumn{1}{c}{ Samples } & Pt & Pd & Ru & Au & Te \\
\hline Pt NTs & $97 \%$ & & & & $3 \%$ \\
PtTe NWs & $58 \%$ & & & & $42 \%$ \\
PtPdTe NWs & $32 \%$ & $34 \%$ & & & $34 \%$ \\
PtAuTe NWs & $21 \%$ & & & $10 \%$ & $69 \%$ \\
PtPdRuTe NTs & $14 \%$ & $37 \%$ & $23 \%$ & & $26 \%$ \\
Pt NPs & $100 \%$ & & & & \\
Pt NFs & $100 \%$ & & & \\
\hline
\end{tabular}


Table S2. Summary of literature catalytic parameters of various Pt-based MOR catalysts.

\begin{tabular}{|c|c|c|c|c|c|}
\hline Catalysts & Electrolytes & $\begin{array}{c}\text { Scan } \\
\text { rates } \\
\left(\mathrm{mV} \mathrm{s}^{-1}\right)\end{array}$ & $\begin{array}{c}\text { Mass } \\
\text { activities } \\
\text { (mA mg }{ }^{-1} \\
\text { Pt) }\end{array}$ & $\begin{array}{c}\text { Specific } \\
\text { activities } \\
\left(\mathbf{m A ~ c m} \mathbf{~ c m}^{-2}\right)\end{array}$ & References \\
\hline Ultrathin Pt NWs & $\begin{array}{c}0.5 \mathrm{M} \mathrm{H}_{2} \mathrm{SO}_{4}+ \\
0.5 \mathrm{M} \mathrm{CH}_{3} \mathrm{OH}\end{array}$ & 20 & $\sim 500$ & $\sim 1.1$ & 5 \\
\hline $\begin{array}{c}\text { Pt multiple- } \\
\text { twinned } \\
\text { nanowire network }\end{array}$ & $\begin{array}{l}0.5 \mathrm{M} \mathrm{H}_{2} \mathrm{SO}_{4}+ \\
1.0 \mathrm{M} \mathrm{CH}_{3} \mathrm{OH}\end{array}$ & 50 & 580.97 & / & 6 \\
\hline $\mathrm{Pt} / \mathrm{OMCS}$ & $\begin{array}{l}0.5 \mathrm{M} \mathrm{H}_{2} \mathrm{SO}_{4}+ \\
1.0 \mathrm{M} \mathrm{CH}_{3} \mathrm{OH}\end{array}$ & 50 & 671 & 0.78 & 7 \\
\hline Pt/graphene & $\begin{array}{c}0.5 \mathrm{M} \mathrm{CH}_{3} \mathrm{OH}+ \\
0.5 \mathrm{M} \mathrm{H}_{2} \mathrm{SO}_{4}\end{array}$ & 50 & 199.6 & l & 8 \\
\hline Pt-NTds & $\begin{array}{l}0.1 \mathrm{M} \mathrm{HClO}_{4}+ \\
1.0 \mathrm{M} \mathrm{CH}_{3} \mathrm{OH}\end{array}$ & 20 & 201.35 & 2.36 & 9 \\
\hline $\mathrm{Pt} / \mathrm{S}-\mathrm{rGO}$ & $\begin{array}{c}0.5 \mathrm{M} \mathrm{H}_{2} \mathrm{SO}_{4}+ \\
1 \mathrm{M} \mathrm{CH}_{3} \mathrm{OH}\end{array}$ & 50 & 465 & l & 10 \\
\hline $\begin{array}{l}\text { Pt nanocube } \\
\text { assemblies }\end{array}$ & $\begin{array}{l}0.1 \mathrm{M} \mathrm{HClO}_{4}+ \\
0.2 \mathrm{M} \mathrm{CH}_{3} \mathrm{OH}\end{array}$ & 50 & 292 & 0.76 & 11 \\
\hline Pt-HNSs & $\begin{array}{l}0.5 \mathrm{M} \mathrm{H}_{2} \mathrm{SO}_{4}+ \\
1.0 \mathrm{M} \mathrm{CH}_{3} \mathrm{OH}\end{array}$ & 50 & 99.3 & 42.5 & 12 \\
\hline $\begin{array}{c}\text { Nanoporous } \\
\text { Platinum } \\
\text { Nanoparticles }\end{array}$ & $\begin{array}{l}0.5 \mathrm{M} \mathrm{H}_{2} \mathrm{SO}_{4}+ \\
1.0 \mathrm{M} \mathrm{CH}_{3} \mathrm{OH}\end{array}$ & 50 & / & 29.84 & 13 \\
\hline $\mathrm{PtNSs} / \mathrm{CNTs} / \mathrm{CP}$ & $\begin{array}{l}0.5 \mathrm{M} \mathrm{H}_{2} \mathrm{SO}_{4}+ \\
1.0 \mathrm{M} \mathrm{CH}_{3} \mathrm{OH}\end{array}$ & 20 & 233.33 & $\sim 60$ & 14 \\
\hline $\mathrm{PtPb} / \mathrm{Pt}$ nanoplate & $\begin{array}{c}0.1 \mathrm{HClO}_{4}+ \\
0.1 \mathrm{M} \mathrm{CH}_{3} \mathrm{OH}\end{array}$ & 50 & 1500 & 2.7 & 15 \\
\hline $\mathrm{Pt}_{3} \mathrm{Co} / \mathrm{C}$ & $\begin{array}{c}0.1 \mathrm{HClO}_{4}+ \\
0.2 \mathrm{M} \mathrm{CH}_{3} \mathrm{OH}\end{array}$ & 50 & 1000 & 1.9 & 16 \\
\hline $\begin{array}{c}\mathrm{Pt}_{27} \mathrm{Au}_{3} \mathrm{Cu}-\mathrm{AA} \\
\mathrm{NTs}\end{array}$ & $\begin{array}{l}0.5 \mathrm{M} \mathrm{H}_{2} \mathrm{SO}_{4}+ \\
1.0 \mathrm{M} \mathrm{CH}_{3} \mathrm{OH}\end{array}$ & 50 & 1698.8 & l & 17 \\
\hline PtPdRuTe NTs & $\begin{array}{l}0.5 \mathrm{M} \mathrm{H}_{2} \mathrm{SO}_{4}+ \\
1.0 \mathrm{M} \mathrm{CH}_{3} \mathrm{OH}\end{array}$ & 50 & 1261.5 & 2.96 & 18 \\
\hline $\begin{array}{c}\text { Ordered Pt NTs } \\
\text { monolayer }\end{array}$ & $\begin{array}{c}0.1 \mathrm{HClO}_{4}+ \\
1.0 \mathrm{M} \mathrm{CH}_{3} \mathrm{OH}\end{array}$ & 50 & 1349 & 2.7 & This work \\
\hline
\end{tabular}


Table S3. Selection of the parameters in MOR.

\begin{tabular}{ccccccccc}
\hline Parameters & $\mathrm{N}$ & $\boldsymbol{\varphi}_{\mathbf{0}}$ & $\boldsymbol{\theta}_{\boldsymbol{e q}}$ & $\alpha$ & $\lambda$ & $\boldsymbol{k}_{\boldsymbol{\eta}=\mathbf{0}}$ & $\boldsymbol{u}_{\mathbf{0}}$ & $\boldsymbol{\varepsilon}$ \\
\hline Values & 100 & 0.5 & 0.01 & 0.15 & 1.0 & 1000 & 1.2 & 6.01 \\
\hline
\end{tabular}

Table S4. Selection of the parameters in MOR of 0D particles.

\begin{tabular}{ccccccccc}
\hline Parameters & $\mathrm{N}$ & $\boldsymbol{\varphi}_{\mathbf{0}}$ & $\boldsymbol{\theta}_{\boldsymbol{e q}}$ & $\alpha$ & $\lambda$ & $\boldsymbol{k}_{\boldsymbol{\eta}=\mathbf{0}}$ & $\boldsymbol{u}_{\mathbf{0}}$ & $\boldsymbol{\varepsilon}$ \\
\hline Values & 100 & 0.5 & 0.01 & 0.15 & 1.0 & 1000 & 1.2 & 6.01 \\
\hline
\end{tabular}

Table S5. Selection of the parameters in MOR of 2D films.

\begin{tabular}{ccccccccc}
\hline Parameters & $\mathrm{N}$ & $\boldsymbol{\varphi}_{\mathbf{0}}$ & $\boldsymbol{\theta}_{\boldsymbol{e q}}$ & $\alpha$ & $\lambda$ & $\boldsymbol{k}_{\boldsymbol{\eta}=\mathbf{0}}$ & $\boldsymbol{u}_{\mathbf{0}}$ & $\boldsymbol{\varepsilon}$ \\
\hline Values & 100 & 0.5 & 0.01 & 0.15 & 1.0 & 1000 & 1.2 & 4.52 \\
\hline
\end{tabular}

Table S6. Selection of the parameters in FAOR.

\begin{tabular}{ccccccccc}
\hline Parameters & $\mathrm{N}$ & $\boldsymbol{\varphi}_{\mathbf{0}}$ & $\boldsymbol{\theta}_{\boldsymbol{e q}}$ & $\alpha$ & $\lambda$ & $\boldsymbol{k}_{\boldsymbol{\eta}=\mathbf{0}}$ & $\boldsymbol{u}_{\mathbf{0}}$ & $\boldsymbol{\varepsilon}$ \\
\hline Values & 100 & 0.8 & 0.02 & 0.15 & 1.0 & 2000 & 1.2 & 6.01 \\
\hline
\end{tabular}

\section{References}

(1) Ma, S. Y.; Li, H. H.; Hu, B. C.; Cheng, X.; Fu, Q. Q.; Yug, S. H., Synthesis of Low Pt-Based Quaternary PtPdRuTe Nanotubes with Optimized Incorporation of Pd for Enhanced Electrocatalytic Activity. J. Am. Chem. Soc. 2017, 139, 5890.

(2) Yan, X. P.; Liu, H. F.; Liew, K. Y., Size Control of Polymer-Stabilized Ruthenium Nanoparticles by Polyol Reduction. J. Mater. Chem. 2001, 11, 3387.

(3) Alayoglu, S.; Nilekar, A. U.; Mavrikakis, M.; Eichhorn, B., Ru-Pt Core-Shell Nanoparticles for Preferential Oxidation of Carbon Monoxide in Hydrogen. Nat. Mater. 2008, 7, 333 .

(4) Hao, Y. F.; Wang, X. D.; Shen, J. F.; Yuan, J. H.; Wang, A. J.; Niu, L.; Huang, S. T., One-Pot Synthesis of Single-Crystal Pt Nanoplates Uniformly Deposited on Reduced 
Graphene Oxide, and Their High Activity and Stability on the Electrocalalytic Oxidation of Methanol. Nanotechnology 2016, 27, 145602.

(5) Xia, B. Y.; Wu, H. B.; Yan, Y.; Lou, X. W.; Wang, X., Ultrathin and Ultralong SingleCrystal Platinum Nanowire Assemblies with Highly Stable Electrocatalytic Activity. $J$. Am. Chem. Soc. 2013, 135, 9480.

(6) Ruan, L.; Zhu, E.; Chen, Y.; Lin, Z.; Huang, X.; Duan, X.; Huang, Y., Biomimetic Synthesis of An Ultrathin Platinum Nanowire Network with a High Twin Density for Enhanced Electrocatalytic Activity and Durability. Angew. Chem. Int. Ed. 2013, 52, 12577.

(7) Zhang, C.; Xu, L.; Shan, N.; Sun, T.; Chen, J.; Yan, Y., Enhanced Electrocatalytic Activity and Durability of $\mathrm{Pt}$ Particles Supported on Ordered Mesoporous Carbonspheres. ACS Catalysis 2014, 4, 1926.

(8) Li, Y.; Tang, L.; Li, J., Preparation and Electrochemical Performance for Methanol Oxidation of Pt/Graphene Nanocomposites. Electrochem. Commun. 2009, 11, 846.

(9) Rana, M.; Chhetri, M.; Loukya, B.; Patil, P. K.; Datta, R.; Gautam, U. K., HighYield Synthesis of Sub-10 nm Pt Nanotetrahedra with Bare 111 Facets for Efficient Electrocatalytic Applications. ACS Appl. Mater. Interfaces 2015, 7, 4998.

(10) Lu, J.; Li, Y.; Li, S.; Jiang, S. P., Self-Assembled Platinum Nanoparticles on Sulfonic Acid-Grafted Graphene as Effective Electrocatalysts for Methanol Oxidation in Direct Methanol Fuel Cells. Sci. Rep. 2016, 6, 21530.

(11) Sun, X.; Zhu, X.; Zhang, N.; Guo, J.; Guo, S.; Huang, X., Controlling and Self assembling of Monodisperse Platinum Nanocubes as Efficient Methanol Oxidation Electrocatalysts. Chem. Commun. (Camb) 2015, 51, 3529.

(12) Yang, M.; Cai, Q.; Liu, C.; Wu, R.; Sun, D.; Chen, Y.; Tang, Y.; Lu, T., Monodispersed Hollow Platinum Nanospheres: Facile Synthesis and Their Enhanced Electrocatalysis for Methanol Oxidation. J. Mater. Chem. A 2014, 2, 13738.

(13) Hussein, H. E. M.; Amari, H.; Macpherson, J. V., Electrochemical Synthesis of Nanoporous Platinum Nanoparticles Using Laser Pulse Heating: Application to Methanol Oxidation. ACS Catalysis 2017, 7, 7388.

(14) Fang, W. C.; Chen, F. R.; Tsai, M. C.; Chou, H. Y.; Wu, H. C.; Hsieh, C. K., 
Electrochemical Deposited High-Crystallinity Vertical Platinum Nanosheets onto the Carbon Nanotubes Directly Grown on Carbon Paper for Methanol Oxidation. Surf. Coat. Technol. 2017, 320, 584.

(15) Bu, L. Z.; Zhang, N.; Guo, S. J.; Zhang, X.; Li, J.; Yao, J. L.; Wu, T.; Lu, G.; Ma, J. Y.; Su, D.; Huang, X., Biaxially Strained PtPb/Pt Core/Shell Nanoplate Boosts Oxygen Reduction Catalysis. Science 2016, 354, 1410.

(16) Wang, P.; Zhang, X.; Zhang, J.; Wan, S.; Guo, S.; Lu, G.; Yao, J.; Huang, X., Precise Tuning in Platinum-Nickel/Nickel Sulfide Interface Nanowires for Synergistic Hydrogen Evolution Catalysis. Nat. Commun. 2017, 8, 14580.

(17) Fu, Q. Q.; Li, H. H.; Ma, S. Y.; Hu, B. C.; Yu, S. H., A Mixed-Solvent Route to Unique PtAuCu Ternary Nanotubes Templated from $\mathrm{Cu}$ Nanowires as Efficient Dual Electrocatalysts. Sci. China Mater. 2016, 59, 112.

(18) Kim, D.; Xie, C. L.; Becknell, N.; Yu, Y.; Karamad, M.; Chan, K.; Crumlin, E. J.; Norskov, J. K.; Yang, P. D., Electrochemical Activation of $\mathrm{CO}_{2}$ through Atomic Ordering Transformations of AuCu Nanoparticles. J. Am. Chem. Soc. 2017, 139, 8329. 